\title{
Triple negative breast carcinoma is a prognostic factor in Taiwanese women
}

\author{
Che Lin ${ }^{1}$, Su-Yu Chien ${ }^{2}$, Li-Sheng Chen ${ }^{1}$, Shou-Jen Kuo ${ }^{1}$, Tsai-Wang Chang*3 \\ and Dar-Ren Chen*1
}

\author{
Address: ${ }^{1}$ Comprehensive Breast Cancer Center, Changhua Christian Hospital, Changhua, Taiwan, ${ }^{2}$ Department of Pharmacology, Changhua \\ Christian Hospital, Changhua, Taiwan and ${ }^{3}$ Department of General Surgery, National Cheng Kung University Hospital, Tainan, Taiwan \\ Email: Che Lin - chelin1chelin1@yahoo.com.tw; Su-Yu Chien - d4605@cch.org.tw; Li-Sheng Chen - 131032@cch.org.tw; Shou- \\ Jen Kuo-40225@cch.org.tw; Tsai-Wang Chang* - twchang@mail.ncku.edu.tw; Dar-Ren Chen* - darren_chen@cch.org.tw \\ * Corresponding authors
}

Published: 18 June 2009

BMC Cancer 2009, 9:192 doi:10.1 186/147|-2407-9-192
Received: 31 October 2008

Accepted: 18 June 2009

This article is available from: http://www.biomedcentral.com//47/-2407/9//92

(C) 2009 Lin et al; licensee BioMed Central Ltd.

This is an Open Access article distributed under the terms of the Creative Commons Attribution License (http://creativecommons.org/licenses/by/2.0), which permits unrestricted use, distribution, and reproduction in any medium, provided the original work is properly cited.

\begin{abstract}
Background: Currently, there is a debate as to whether triple negative breast carcinoma (TNBC) has a worse prognosis than non-TNBC. Our aim was to determine whether TNBC is a prognostic factor for survival.

Methods: We identified I,048 Taiwanese breast carcinoma patients, of whom 167 (15.9\%) had TNBC. Data used for analysis were derived from our cancer registry database for women with breast cancer who were diagnosed between 2002 January and 2006 December.

Results: In the Kaplan-Meier analysis, tumor subgroup (TNBC vs. non-TNBC) was a prognosis factor related to 5-year overall survival. In the univariate analysis, tumor subgroup (TNBC vs. nonTNBC) was a significant factor related to 5-year overall survival, in addition to age, tumor size, lymph node, metastasis, grade, stage, estrogen receptor status, progesterone receptor status, and HER2 overexpression status. In the multivariate analysis, tumor subgroup was not a significant factor related to 5-year disease-free survival (DFS). In node-positive patients, tumor subgroup was a significant factor related to 5-year overall survival, in addition to age, tumor size, metastasis, and grade. In node-negative patients, tumor subgroup was not a significant factor related to 5-year disease-free survival and 5-year overall survival.
\end{abstract}

Conclusion: Our results indicated that TNBC patients in Taiwan have worse 5-year overall survival than non-TNBC patients. Notably, in node-positive patients, TNBC played a prognostic role in 5-year overall survival.

\section{Background}

Many oncologists think breast cancer is a clinically heterogeneous disease with different responses to treatment and outcomes $[1,2]$. Sixty to $80 \%$ of tumors are positive for the estrogen receptor (ER) and/or progesterone (PgR), and $20 \%$ to $40 \%$ have her $2 /$ neu (HER2) gene over-expres- sion [1]. Interestingly, some recent data suggested that triple negative breast carcinomas (TNBC), ER-negative, PgRnegative and HER2-negative exhibited different clinical outcomes $[3,4]$. However, there is uncertainty about the appropriate survival role for TNBC. Information on the TNBC subtype is still limited and confusing in adjuvant 
chemotherapies [4-15]. Liedtke revealed that patients with TNBC have increased pathologic complete response rates (pCR) compared with non-TNBC patients, and those with pCR have excellent survival [3]. Liedtke also demonstrated that patients with residual disease after neoadjuvant chemotherapy have significantly worse survival if they have TNBC compared with non-TNBC, particularly in the first 3 years. Because these studies were done in the other countries, their findings might not apply to Taiwan. In this study, we sought to determine the risk associated with TNBC in Taiwan.

Therefore, the aim of this study was to determine the prognostic significance of TNBC with respect to disease-free survival (DFS) and overall survival in a group of homogeneously-treated Taiwanese breast carcinoma patients.

\section{Methods}

Patients were identified from the databases of the cancer registry at Changhua Christian Hospital, which is located in central Taiwan. Data collection for cancer in this medical center began in 1986 and continued until 2009. The well-trained case managers used the registry software and collected uniform information about all patients with breast cancer who had been examined at least once as outpatients or inpatients in the daily clinical service. This study was approved by the institutional review board of Changhua Christian Hospital (IRB number: 080325). The baseline data included demographic characteristics (e.g., age), tumor characteristics (e.g., tumor size, positivity of lymph node, metastasis, grade, pathologic stage, ER/PgR/ HER2 information and histology). Patients with ductal carcinoma in situ only were excluded. The data underwent a variety of editing checks and procedures, so as to omit duplicate records. The quality of the cancer registry database was reviewed and approved by the committee, which consisted of radiologists, oncologists, pathologists, surgeons and epidemiologists with special expertise in breast cancer.

Tumor size was determined on the basis of pathological reports from the Changhua Christian Hospital. The Bloom-Richardson grading system was used for tumor grading. This grading scheme is based on three morphologic features: degree of tumor tubule formation, tumor mitotic activity, and nuclear pleomorphism of tumor cells. Seven possible scores are condensed into three Bloom-Richardson grades: I, II, or III. Staging in this study was presented by the American Joint Committee on Cancer stage group.

Immunohistochemistry (IHC) analysis was performed on formalin-fixed, paraffin-embedded breast cancer tissue. The ER and PgR analysis was based on a IHC assay, in which a report of $10 \%$ or greater of cells that had nuclear staining for ER was considered a positive result as well as PgR. IHC was performed with anti-ER (NeoMarkers, clone: SP1, dilution: 1:200, Fermont, California) and antiPgR antibody (NeoMarkers, clone:SP2, dilution: 1:250, Fermont, California) by an autostaining system (Ventana Medical Systems, Tucson, Arizona).

Breast cancer tumors were classified as HER2-positive if they demonstrated HER2 gene amplification using the fluorescence in-situ hybridization method, or were scored as $3+$ by an IHC method. HER2 IHC only used cell membrane localization to interpret (Dako, Carpinteria, California). The intensity of the membrane staining was defined by a semiquantitative score ( 0 to $3+)$. Tumor staining was compared to staining of normal breast epithelium from the same patient as a negative control. For clinical purposes, 3+ staining, defined as uniform and intense membrane staining in more than $30 \%$ of invasive breast cancer cells, was considered overexpression. No staining or weak incomplete membrane staining was considered a negative result.

Data used for analysis were derived from the cancer registry database of women with breast cancer who were diagnosed between 2002 January and 2006 December. Data for analysis started from 2002, because there was a lack of information on HER2 in patients before 2002. DFS was defined as freedom from breast cancer recurrence or breast death. Overall survival was defined as freedom from breast cancer death or other causes of death.

Postoperative adjuvant therapy has been performed based on the recommendation of NCCN or St. Gallen guidelines with anthracycline-based regimens (in this study mostly FEC therapy: 5-FU $500 \mathrm{mg} / \mathrm{m}^{2}$, epirubicin $75-90 \mathrm{mg} / \mathrm{m}^{2}$, cyclophosphamide $500 \mathrm{mg} / \mathrm{m}^{2}$ ) as chemotherapy. Taxanes (paclitaxel, docetaxel) were added to follow FEC therapy in few high risk patients.

The patient group included 1,048 females with an average age of 51.8 years (standard deviation $[\mathrm{SD}]=11.9$ years). Data are expressed as mean \pm SD for continuous variables. Independent $t$ tests were used for the comparison of continuous variables. Categorical variables were normally tested by the $\chi^{2}$ test when appropriate. All $p$ values are two-tailed; a $p$ value of less than 0.05 was considered to indicate statistical significance.

We used Cox proportional hazard analysis to assess the risk of recurrence or mortality relative to the prognostic factors in breast cancer cases. Cumulative survival rates of breast cancer cases were analyzed by the Kaplan-Meier method. The differences of cumulative survival were assessed using the log-rank method. All statistical analyses were performed with SAS 9.1 software. 


\section{Results}

Eighty-two (7.8\%) patients died for cancer-related reasons during their follow-up, up to 31, December 2007. Five $(0.48 \%)$ patients died for non-cancer-related reasons. The average follow-up time was approximately 40 months. One hundred and sixty-seven patients (15.9\%) had TNBC and the remaining 881 patients ( $84.1 \%)$ were defined as non-TNBC. There was no distributional difference of adverse prognostic factors between the two groups, except lymph node, and grade (Table 1). Seventy-one cases with recurrence of breast cancer were considered as the events.

Table I: Descriptive statistics of women with breast cancer according to tumor subgroup

\begin{tabular}{|c|c|c|c|c|c|}
\hline \multirow[t]{2}{*}{ Features } & & \multirow{2}{*}{$\begin{array}{c}\text { Total } \\
\text { (n) }\end{array}$} & \multicolumn{2}{|c|}{ Tumor subgroup } & \multirow[t]{2}{*}{$p$-value } \\
\hline & & & $\begin{array}{c}\text { TNBC } \\
(n=167)\end{array}$ & $\begin{array}{c}\text { non-TNBC } \\
(\mathrm{n}=88 \mathrm{I})\end{array}$ & \\
\hline Age, years (SD) & & & $51.97(13.04)$ & $51.73(11.79)$ & 0.81 \\
\hline \multicolumn{6}{|c|}{ Tumor size, $\mathrm{cm}(\%)$} \\
\hline & $<2.0$ & 446 & 46.1 & 46.3 & 0.9651 \\
\hline & $\geq 2.0$ & 517 & 53.9 & 53.7 & \\
\hline & Other or Unknown & 85 & & & \\
\hline \multicolumn{6}{|l|}{ Lymph node (\%) } \\
\hline & Negative & 553 & 70.1 & 54.8 & $0.0004^{*}$ \\
\hline & Positive & 413 & 29.9 & 45.2 & \\
\hline & Other or Unknown & 82 & & & \\
\hline \multicolumn{6}{|c|}{ Number of axillary lymph nodes involved (n) } \\
\hline & $1-3$ & 210 & 45.7 & 51.8 & 0.6848 \\
\hline & $4-9$ & 122 & 34.8 & 29.0 & \\
\hline & $10+$ & 79 & 19.6 & 19.2 & \\
\hline Average numbe & xillary lymph nodes involved per node-positive patient & & $6.17(5.91)$ & $6.19(7.92)$ & 0.99 \\
\hline \multicolumn{6}{|c|}{ Metastasis (\%) } \\
\hline & No & 938 & 96.8 & 97.8 & 0.4781 \\
\hline & Yes & 23 & 3.2 & 2.2 & \\
\hline & Unknown & 87 & & & \\
\hline \multicolumn{6}{|c|}{ Metastasis site $(\mathrm{n})$} \\
\hline & Bone & 9 & I & 8 & \\
\hline & Lung & 1 & I & 0 & \\
\hline & Liver & 2 & 0 & 2 & \\
\hline & Other & 2 & 0 & 2 & \\
\hline & Multiple & 5 & I & 4 & \\
\hline & Unknown & 4 & 2 & 2 & \\
\hline \multicolumn{6}{|l|}{ Grade (\%) } \\
\hline & I $\sim 1 \mid$ & 693 & 43.4 & 73.8 & $<0.0001$ \\
\hline & III & 311 & 56.6 & 26.2 & \\
\hline & Unknown & 44 & & & \\
\hline \multicolumn{6}{|l|}{ Stage } \\
\hline & $<\|$ & 315 & 35.1 & 32.7 & 0.5622 \\
\hline & $\geq \mathrm{II}$ & 638 & 64.9 & 67.3 & \\
\hline & Unknown & 95 & & & \\
\hline \multicolumn{6}{|c|}{ Estrogen receptor (ER) } \\
\hline & Negative & 369 & 100 & 23 & \\
\hline & Positive & 675 & 0 & 77 & \\
\hline \multicolumn{6}{|c|}{ Progesterone receptor (PR) } \\
\hline & Negative & 383 & 100 & 25 & \\
\hline & Positive & 661 & 0 & 75 & \\
\hline \multicolumn{6}{|c|}{ Her2/neu gene (HER2) overexpression } \\
\hline & Negative & 789 & 100 & 71 & \\
\hline & Positive & 259 & 0 & 29 & \\
\hline \multicolumn{6}{|c|}{ Histological types } \\
\hline & Infiltrating duct carcinoma, NOS & 939 & 91.6 & 89.2 & \\
\hline & Lobular Carcinoma, NOS & 24 & 0 & 2.7 & \\
\hline & Mucinous adenocarcinoma & 24 & 0 & 2.7 & \\
\hline & Infiltrating duct mixed with other types of carcinoma & 18 & 1.8 & 1.7 & \\
\hline & Others & 43 & 6.6 & 3.6 & \\
\hline
\end{tabular}


The univariate analysis for prognostic factors associated with 5-year DFS revealed that the tumor group, whether TNBC or non-TNBC, as well as age and menopausal status, was not statistically significant (Table 2). The univariate analysis for prognostic factors associated with 5-year overall survival revealed that the tumor group as TNBC or non-TNBC was statistically significant, in addition to age, tumor size, lymph node, metastasis, grade, stage, ER status, and PgR status (Table 2).

The multivariate analysis for prognostic factors associated with 5-year DFS revealed that the tumor group as TNBC or non-TNBC was not statistically significant. The multivariate analysis for prognostic factors associated with 5-year overall survival revealed that the tumor group as TNBC or non-TNBC was not statistically significant (Table 3).

Survival curves are shown in the figures. Figure 1 reveals that TNBC tends to display a worse 5 -year overall survival ( $p=0.0026)$ than non-TNBC, using by log-rank analysis. ER-positive and/or PgR-positive and HER2-negative patients had the best clinical outcome, with a 5 -year DFS of $80 \%$; ER-negative, PgR-negative and HER2-positive patients (HER2-positive subtype) showed the worst outcome with a 5 -year DFS of $45 \%$ (Figure 2A). TNBC has relatively poor prognosis. Figure 3A shows ER-positive and/ or PgR-positive and HER2-negative patients had the best clinical outcome, with a 5-year overall survival of $91 \%$, and the HER2-positive subtype showed the worst outcome, with a 5 -year overall survival of 59\%.
To investigate the lymph node factor, we analyzed nodepositive and node-negative patients individually. For node-positive patients, Figure 2B demonstrates that TNBC breast carcinoma tends to show a worse 5-year DFS, by log-rank analysis, and that ER-positive and/or PgR-positive and HER2-negative patients have the best 5-year DFS $(\mathrm{p}=0.0088)$. For node-negative patients, Figure 2C reveals that the four subgroups do not show a 5-year DFS difference, using log-rank analysis $(\mathrm{p}=0.0720)$.

For node-positive patients, Figure $3 \mathrm{~B}$ points out that TNBC breast carcinoma patients tend to have the worst 5 year overall survival, by log-rank analysis, and that ERpositive and/or PgR-positive and HER2-negative patients have the best 5 -year overall survival $(p<0.0001)$. For node-negative patients, Figure 3C indicates that the four subgroups do not show a 5 -year overall survival difference, by log-rank analysis $(p=0.8620)$.

\section{Discussion}

We present the results of the largest Taiwanese study to date that thoroughly investigates the clinical phenotype of TNBC with regard to DFS and overall survival.

Traditionally, breast carcinomas have been classified as hormone receptor-positive or negative. Recently, newer approaches to breast carcinoma classification using geneexpression profiles and IHC biomarkers have identified at least four subtypes [10]. These subtypes are, luminal A (ER-positive and/or PgR-positive and HER2-negative),

Table 2: Prognostic factors for 5-year disease-free survival (DFS) and overall survival in univariate Cox regression analysis

\begin{tabular}{|c|c|c|c|c|c|c|}
\hline \multirow[t]{2}{*}{ Features } & \multicolumn{3}{|c|}{ DFS } & \multicolumn{3}{|c|}{ Overall Survival } \\
\hline & $H R$ & $95 \% \mathrm{Cl}$ & $p$-Value & $H R$ & $95 \% \mathrm{Cl}$ & $p$-Value \\
\hline Age & 1.00 & $0.99-1.01$ & 0.9859 & 1.03 & $1.01-1.04$ & 0.0018 \\
\hline \multicolumn{7}{|l|}{ Menopausal status } \\
\hline (Postmenopausal vs. Premenopausal) & 1.04 & $0.76-1.42$ & 0.8132 & 1.51 & $0.98-2.33$ & 0.0623 \\
\hline Tumor size, $\mathrm{cm}$ & 1.22 & $1.16-1.29$ & $<0.0001$ & 1.20 & $|| 1-.|.3|$ & $<0.0001$ \\
\hline \multicolumn{7}{|l|}{ Lymph node } \\
\hline (Positive vs. Negative) & 3.08 & $2.14-4.42$ & $<0.0001$ & 2.83 & $1.71-4.68$ & $<0.0001$ \\
\hline \multicolumn{7}{|l|}{ Metastasis } \\
\hline (Yes vs. No) & 4.25 & $2.22-8.11$ & $<0.0001$ & 9.40 & $4.78-18.49$ & $<0.0001$ \\
\hline \multicolumn{7}{|l|}{ Grade } \\
\hline (III vs. I II) & 1.71 & $1.23-2.38$ & 0.0014 & 2.03 & $1.31-3.16$ & 0.0017 \\
\hline \multicolumn{7}{|l|}{ Stage } \\
\hline ( $\geq \mathrm{II}$ vs. $<\mathrm{II})$ & 3.03 & $1.87-4.94$ & $<0.0001$ & 2.53 & $1.33-4.84$ & 0.0048 \\
\hline \multicolumn{7}{|l|}{ ER status } \\
\hline (Negative vs. Positive) & 2.26 & $1.64-3.12$ & $<0.0001$ & 3.08 & $1.98-4.79$ & $<0.0001$ \\
\hline \multicolumn{7}{|l|}{ PgR status } \\
\hline (Negative vs. Positive) & 1.95 & $1.40-2.70$ & $<0.0001$ & 2.35 & $1.50-3.68$ & 0.0002 \\
\hline \multicolumn{7}{|l|}{ HER2 overexpression status } \\
\hline (Negative vs. Positive) & 1.97 & $1.4 \mathrm{I}-2.75$ & $<0.0001$ & 1.56 & $0.99-2.46$ & 0.058 \\
\hline \multicolumn{7}{|l|}{ Tumor subgroups } \\
\hline (TNBC vs. non-TNBC) & 1.43 & $0.99-2.06$ & 0.0570 & 1.99 & $1.26-3.13$ & 0.0031 \\
\hline
\end{tabular}


Table 3: Prognostic factors for 5-year DFS and overall survival in multivariate Cox regression analysis

\begin{tabular}{|c|c|c|c|c|c|c|}
\hline \multirow[t]{2}{*}{ Features } & \multicolumn{3}{|c|}{ DFS } & \multicolumn{3}{|c|}{ Overall Survival } \\
\hline & $H R$ & $95 \% \mathrm{Cl}$ & $p$-Value & $H R$ & $95 \% \mathrm{Cl}$ & $p$-Value \\
\hline Age & 1.00 & $0.98-1.01$ & 0.9058 & 1.04 & $1.01-1.06$ & 0.0013 \\
\hline Tumor size, $\mathrm{cm}$ & 1.19 & $1.10-1.28$ & $<0.0001$ & 1.17 & $1.04-1.32$ & 0.0110 \\
\hline \multicolumn{7}{|l|}{ Lymph node } \\
\hline (Positive vs. Negative) & 2.65 & $1.79-3.92$ & $<0.0001$ & 2.36 & $1.35-4.12$ & 0.0026 \\
\hline \multicolumn{7}{|l|}{ Metastasis } \\
\hline (Yes vs. No) & 2.30 & $1.15-4.64$ & 0.0193 & 6.63 & $3.13-14.09$ & $<0.0001$ \\
\hline \multicolumn{7}{|l|}{ Grade } \\
\hline (III vs. I II) & 1.54 & $1.05-2.26$ & 0.0269 & 2.11 & $1.23-3.62$ & 0.0065 \\
\hline \multicolumn{7}{|l|}{ Tumor subgroups } \\
\hline (TNBC vs. non-TNBC) & 1.28 & $0.82-1.99$ & 0.2835 & 1.66 & $0.93-2.96$ & 0.0881 \\
\hline \multicolumn{7}{|l|}{ Node-positive } \\
\hline Age & 0.99 & $0.97-1.01$ & 0.2960 & 1.04 & $1.01-1.07$ & 0.0161 \\
\hline Tumor size, $\mathrm{cm}$ & 1.23 & $1.10-1.37$ & 0.0002 & 1.29 & $|| 1-.|.5|$ & 0.0011 \\
\hline \multicolumn{7}{|l|}{ Metastasis } \\
\hline (Yes vs. No) & 2.37 & $1.15-4.88$ & 0.0198 & 7.52 & $3.37-16.79$ & $<0.0001$ \\
\hline \multicolumn{7}{|l|}{ Grade } \\
\hline (III vs. I II) & 1.49 & $0.93-2.36$ & 0.0948 & 2.46 & $1.23-4.90$ & 0.0109 \\
\hline \multicolumn{7}{|l|}{ Tumor subgroups } \\
\hline (TNBC vs. non-TNBC) & 1.13 & $0.63-2.03$ & 0.6847 & 2.20 & $1.08-4.49$ & 0.0296 \\
\hline \multicolumn{7}{|l|}{ Node-negative } \\
\hline Age & 1.02 & $0.99-1.04$ & 0.1801 & 1.04 & $1.01-1.07$ & 0.0186 \\
\hline Tumor size, $\mathrm{cm}$ & 1.16 & $|.02-| .3 \mid$ & 0.0236 & 1.00 & $0.74-1.36$ & 0.9813 \\
\hline \multicolumn{7}{|l|}{ Metastasis* } \\
\hline (Yes vs. No) & - & - & - & - & - & - \\
\hline \multicolumn{7}{|l|}{ Grade } \\
\hline (III vs. I II) & 1.49 & $0.75-2.94$ & 0.2526 & 1.69 & $0.69-4.12$ & 0.2525 \\
\hline \multicolumn{7}{|l|}{ Tumor subgroups } \\
\hline (TNBC vs. non-TNBC) & 1.52 & $0.75-3.06$ & 0.2461 & 1.28 & $0.47-3.19$ & 0.6815 \\
\hline
\end{tabular}

* Patients without metastasis were censor observations by the end of study period

luminal B (ER-positive and/or PgR-positive and HER2positive), basal-like (ER-negative, PgR-negative and HER2-negative; mostly TNBC) and HER2-positive (ERnegative, PgR-negative and HER2-positive). ER- and PgRnegative tumors are generally thought to have a poor prognosis because of a deficiency of hormone therapy strategies. HER2-negative tumors lack the benefit of HER2-targeted therapy and are thought to imply a worse prognosis, as well [10]; however, few TNBC data have been reported among non-Western populations. Our data showed that TNBC in Taiwan may have a different meaning than in Western countries.

Table 1 revealed TNBC subgroup had higher rates of nodenegative cases, in agreement with comparable studies $[4,12,16,17]$. Some series had different results from ours $[3,6,7,10,13,14,18,19]$.
Our univariate Cox regression analysis demonstrated that tumor size, lymph node status, metastasis, grade, stage, ER status, PgR status, and HER2 status except tumor TNBC subgroup were the prognostic factors for 5-year DFS, in contrast to comparable series $[4,6,17]$. There were discrepant findings between the outcomes of DFS and overall survival in our data. In CALGB 9344 trial, taxanes demonstrated a statistically significant improvement in DFS but not overall survival. It may interpret the discrepancy. In this study the chemotherapy regimen of most patients was FEC therapy. Due to Taiwan national health insurance policy, using FEC followed by Taxanes was only allowed in node-positive and ER negative breast cancer women. In Taiwan node-positive TNBC patients were allowed to receive Taxanes which improved DFS but not overall survival. Furthermore, Yin et al showed $85.1 \%$ of their patients were administered adjuvant chemotherapy 


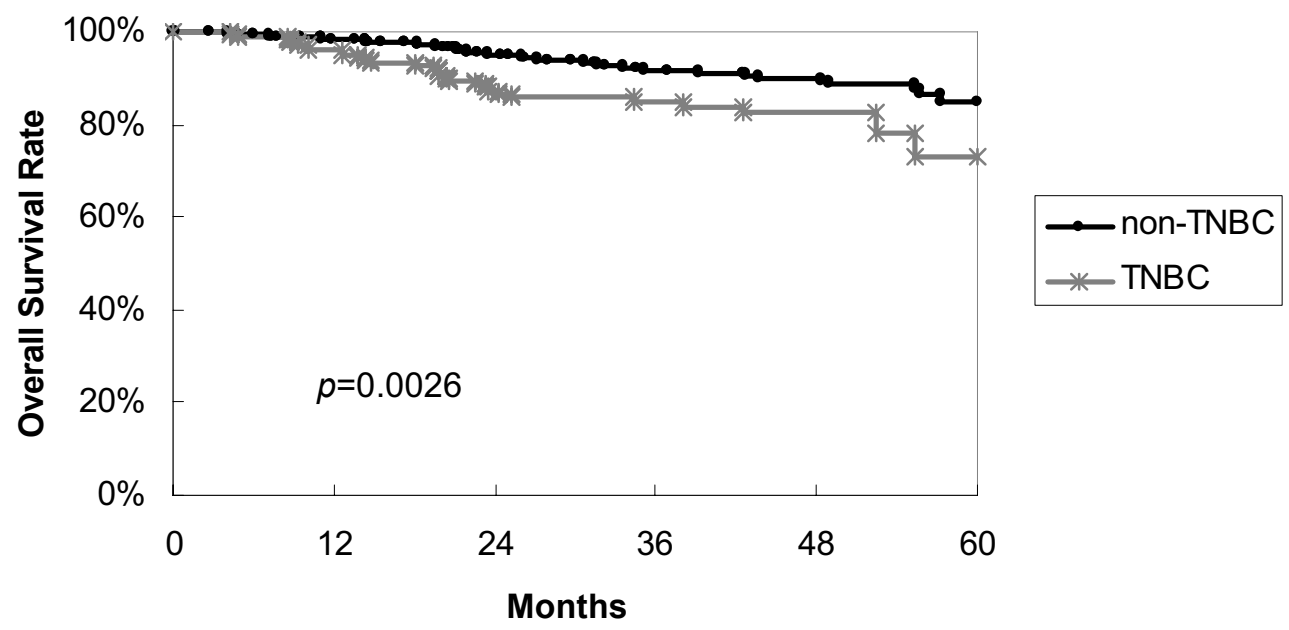

\section{Figure I}

Overall survival curves by tumor subgroup. Triple negative breast cancer (TNBC) tended to display a worse 5-year overall survival $(p=0.0026)$ than non-TNBC, by log-rank analysis.

of different regimens for 4-6 cycles [4]. Mersin said their patients had adjuvant chemotherapy according to the current guidelines at that time [6]. Fulford et al. reported DFS and overall survival results that were different from ours. Their study demonstrated that basal tumors, mostly TNBC, exhibited a significantly better DFS and overall survival than non-basal tumors; however, the study was based on 470 patients with grade III invasive ductal carcinomas diagnosed between 1975 and 1991. Twenty six percent of the patients received adjuvant chemotherapy [17].

Our multivariate Cox regression analysis demonstrated that tumor subgroup (TNBC vs. non-TNBC) was not the prognostic factor for 5-year overall survival These findings were in contrast with those of previous studies $[3,5,12$ $14,20]$. Liedtke reported that decreased 3-year overall survival was observed for patients with TNBC, compared with non-TNBC (3-year overall survival rates: $74 \%$ vs. $89 \% ; \mathrm{HR}=2.53 ; 95 \% \mathrm{CI}, 1.77$ to $3.57 ; p<0.001$ ) [3]. In addition, our results are consistent with those from other studies with respect to prognostic factors for overall survival [16]. Kim reported that basal-like carcinoma, mostly TNBC, did not show survival difference between other subgroups, except the HER2-positive subgroup.

To further study the lymph node factor, we stratified patients into node-positive and node-negative groups. In node-positive patients, TNBC breast carcinoma tended to demonstrate the worst 5-year overall survival by log-rank analysis (Figure 3B), and ER-positive and/or PgR-positive and HER2-negative patients had the best 5-year overall survival $(p<0.0001)$. Figure 3C shows four subgroups of node-negative patients that did not reveal a 5-year overall survival difference by log-rank analysis $(p=0.8620)$. Our results are in contrast to those of a previous study by Rakha, which reported that both univariate and multivariate analyses showed basal phenotype, mostly TNBC, was the only significant and independent prognostic marker in the node-negative patients [10].

Contrary to our supposition, our data in multivariate analysis showed that tumor subgroup (TNBC vs. nonTNBC) was not a prognostic factor for DFS. This study also revealed ER-positive and/or PgR-positive and HER2-negative patients had the best 5-year DFS, and the HER2-positive subtype showed the worst 5-year DFS. These findings were consistent with those of a previous study [6]. Meanwhile, our results contrast with those from other studies in terms of prognostic factors for DFS [3,4,12-14,20]. Liedtke reported that in multivariate analysis, a significantly decreased progression-free survival was observed for patients with TNBC compared with non-TNBC at the seventh year [3]. Yin reported that in multivariate analysis, TNBC had a significantly increased likelihood of recurrence within 2 years after surgery rather than thereafter [4]. Recent studies demonstrated that TNBC tends toward distant metastasis to the bone, soft tissue and viscera $[3,10]$. Liedtke reported that TNBC has a higher predilection for visceral metastasis and early recurrence within the first 3 years of follow-up [3]. Statistically, our study did not substantiate a higher predilection of TNBC factor for metastasis. In this study, there was no difference in DFS and a significant difference in overall survival in univariate analysis. This might indicate the difference in overall survival after recurrence. In our study, treatment 
A

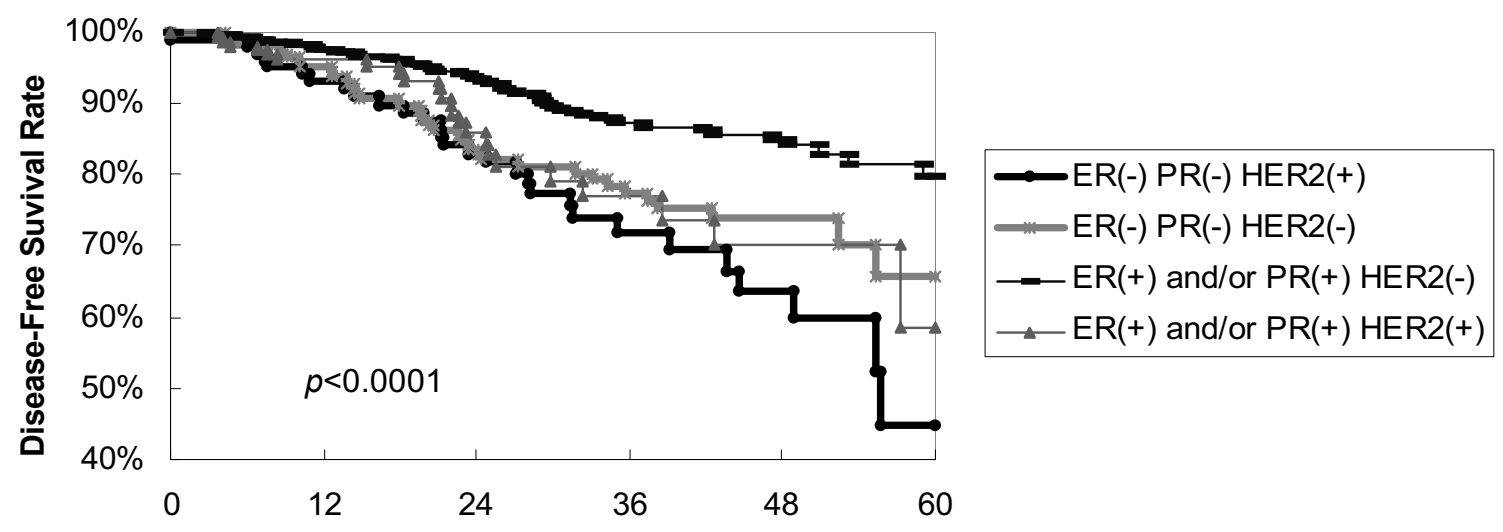

Months

B.

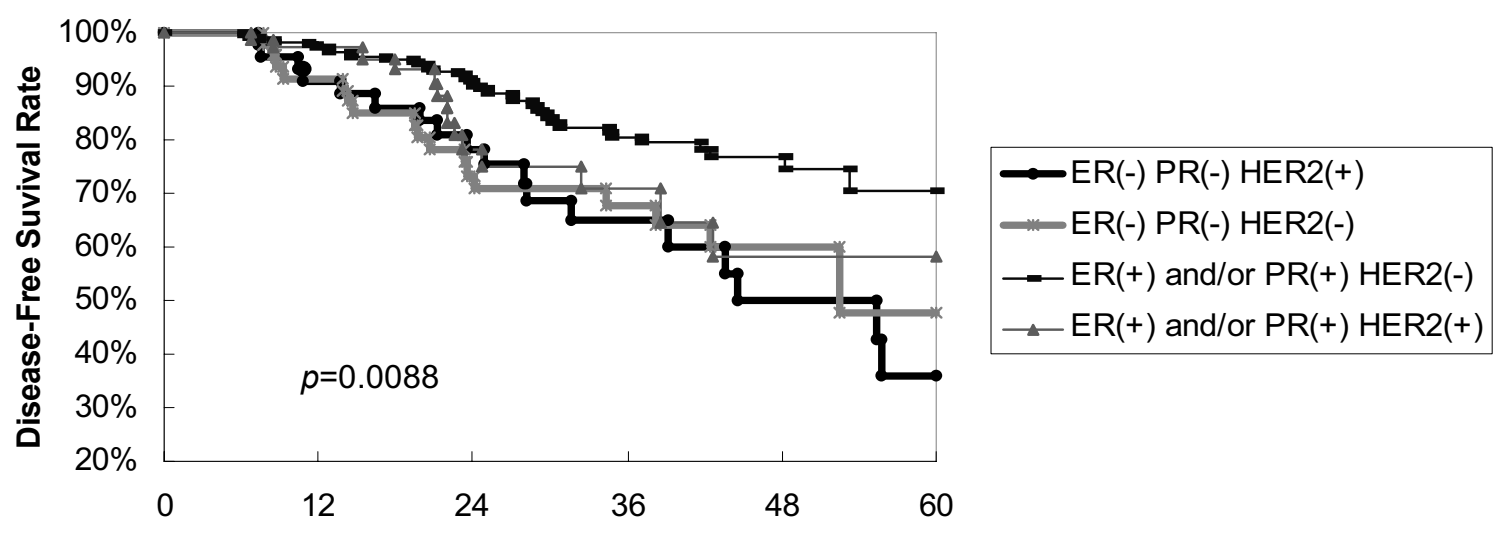

Months

C.

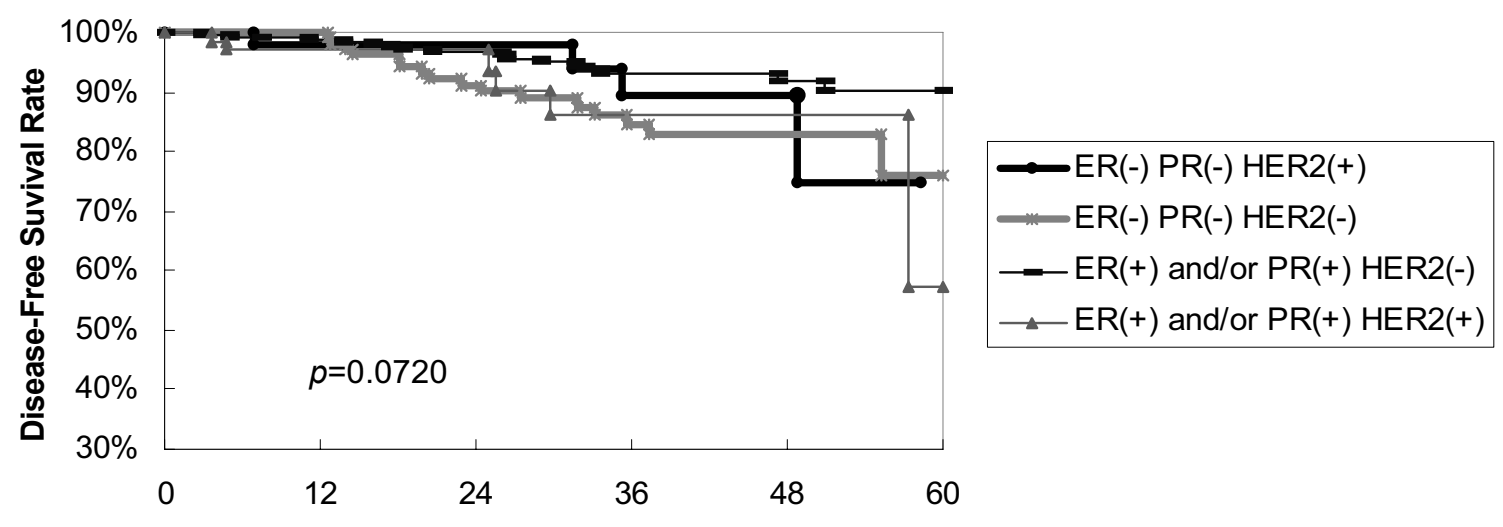

Months

Figure 2

Disease-free survival (DFS) curves according to patient group. A, among I,048 patients, ER-negative and PgR-negative and HER2-positive breast carcinoma tended to display the worst 5-year DFS, by log-rank analysis; ER-positive and/or PgR-positive and HER2-negative patients had the best 5-year overall survival; $p<0.000 \mathrm{I}$. B, in the node-positive patients, TNBC tended to display a worse 5-year DFS by log-rank analysis; ER-positive and/or PgR-positive and HER2-negative patients had the best 5-year DFS; $p=0.0088$. C, in the node-negative patients, four subgroups showed no 5-year DFS difference by log-rank analysis; $p=0.0720$. We followed up the node-negative HER2-positive patients up to 48 months. ER, estrogen receptor; PgR, progesterone receptor; HER2, her2/neu gene over-expression. 
A.

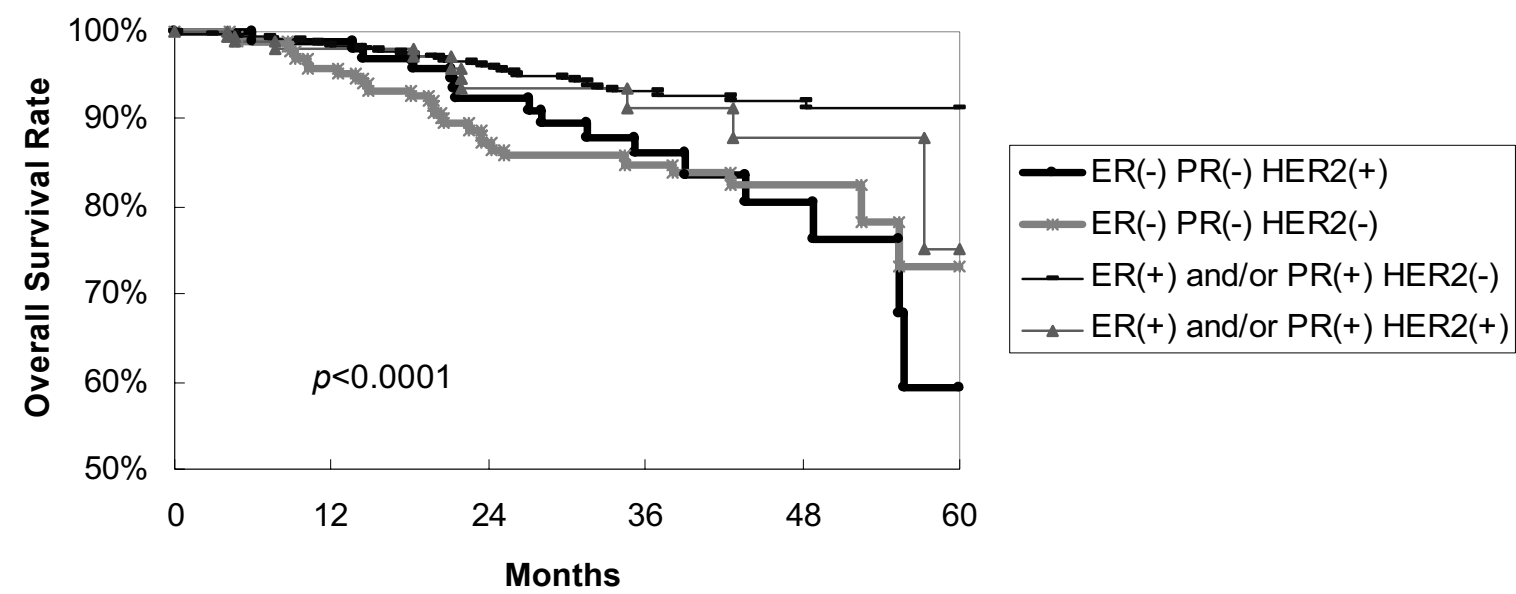

B.

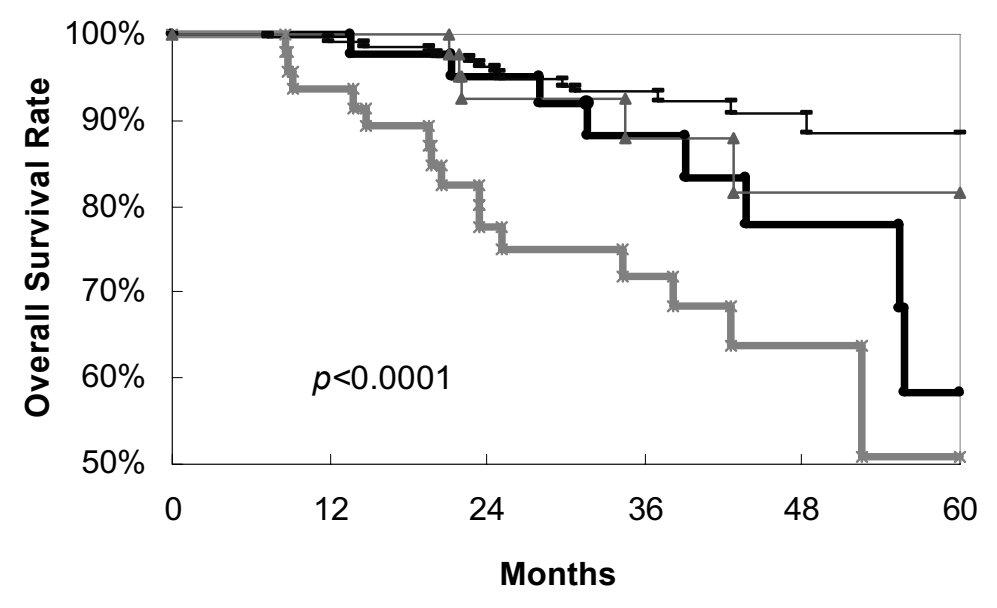

C.

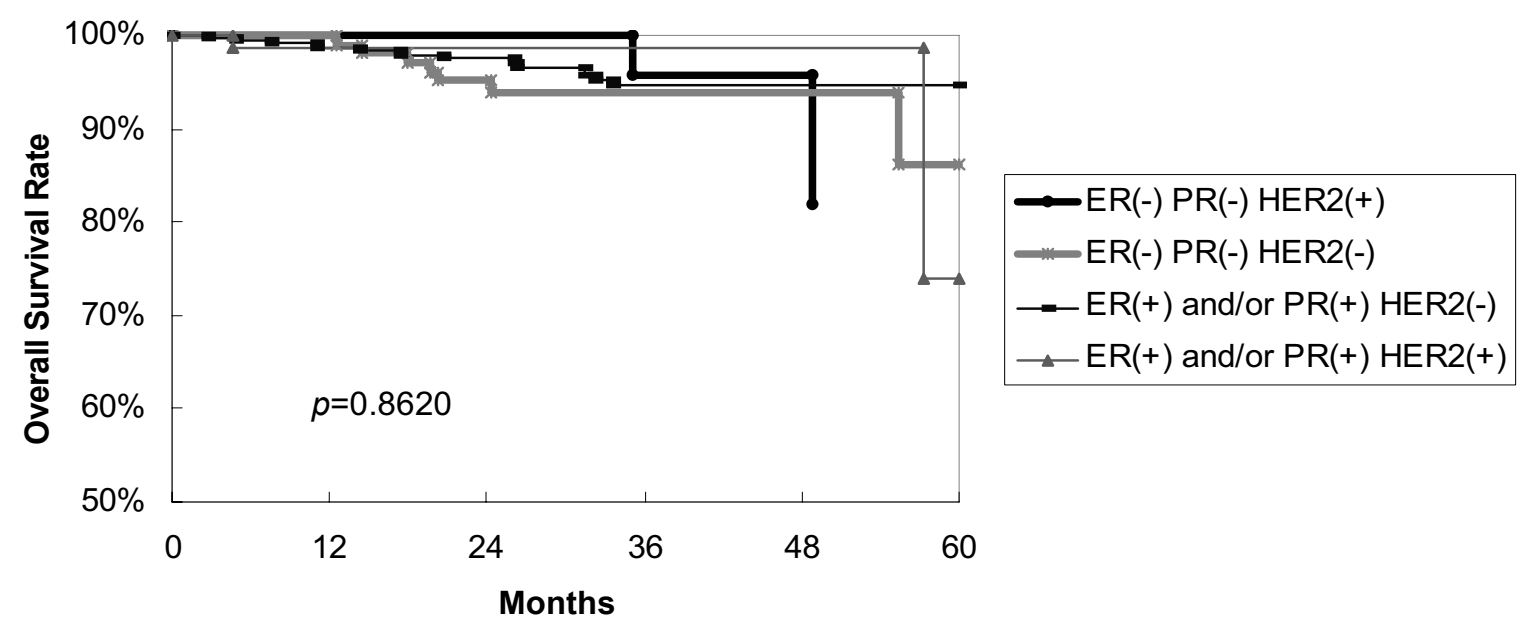

Figure 3

Overall survival curves according to patient group. A, among I,048 patients, HER2-positive breast carcinoma tended to display the worst 5-year overall survival by log-rank analysis; our ER-positive and/or PgR-positive and HER2-negative patients had the best 5 -year DFS; $p<0.000$ I. B, in the node-positive patients, TNBC tended to demonstrate the worst 5 -year overall survival by log-rank analysis; ER-positive and/or PgR-positive and HER2-negative patients had the best 5-year overall survival; $p$ $<0.000 \mathrm{I}$. C, in the node-negative patients, four subgroups showed no 5 -year overall survival difference, by log-rank analysis; $p$ $=0.8620$. We followed up the node-negative HER2-positive patients up to 48 months. 
after recurrence was based on NCCN or St. Gallen guidelines and multidisciplinary care discussions. Most patients who have a recurrence after breast conservation therapy were given completion mastectomy and adjuvant systemic chemotherapy.

In node-positive patients, our data supported the tendency of TNBC breast carcinoma to show a worse 5-year DFS, by log-rank analysis (Figure 2B). These results contrast with those from a previous study [10]. In node-negative patients, our results revealed that the four subgroups showed no difference in 5-year DFS or in 5-year overall survival. However, these results contrast with those from previous studies $[10,21]$. Rhee in Korea demonstrated that triple-negativity was an independent predictor of shorter relapse-free survival [21]. Table 1 in this study demonstrates that TNBC had a higher ratio of lymph node-negative patients than non-TNBC. This may explain why sometimes in our analysis the TNBC group was better than the HER2-positive subgroup.

However, the heterogeneity of TNBC, follow-up time, case number and other limitations may also explain the differences between our conclusions and those of previous studies. Research into the heterogeneity of TNBC, new neoadjuvant regimens and more molecular-based TNBC classification studies could give us more information about the optimal therapies for TNBC subgroups. Liedtke et al. followed their patients to the 7th year, Yin to the 11 th year, and Cheang to the 15 th years [3-5]. A longer follow-up period may yield different results.

In our study, the TNBC rate was $15.9 \%$, which is similar to reports from Korea and Carolina $[16,18]$, but lower than other reports $[3-6,12,21]$. The race factor could contribute to the difference. Carey also reported that the TNBC subtype has a higher incidence in pre-menopausal African-American women (39\%), compared to non-African-American women of any age (16\%) and post-menopausal African-American women (14\%) [18].

This study revealed TNBC and non-TNBC patients displayed a similar distribution of clinico-pathological characteristics, such as age, tumor size, metastasis and stage, in contrast to other Western studies $[3,10,15,19]$. These previous reports indicated that TNBC was associated with a larger size, and therefore had a poorer outcome than nonTNBC in terms of DFS and overall survival. In our study, overall survival rates between these groups were statistically different in univariate analysis. This result may be related to lymph node, as mentioned. On the other hand, a higher grade was observed in our TNBC patients. In spite of this finding, the DFS for the TNBC subgroup was not statistically different from that of the non-TNBC subgroup, in contrast to earlier studies [3,6,17].
In our study, all of the data inferred that HER2 status added prognostic information for hormone receptor-positive breast cancer patients, confirming that luminal B tumors constituted a poor outcome compared to luminal A tumors, in accordance with other studies $[5,6,22]$. In Taiwan, adjuvant trastuzumab targeted therapy was rarely used before 2006 in the HER2-positive subtype, due to the medical insurance policy, which may have contributed substantially to the worst overall survival.

Some research about epidermal growth factor receptor (EGFR) is interesting, and may provide another possible answer for the question of TNBC prognostic value $[5,11,23]$. Cheang reported that the expanded surrogate of PgR, HER2, EGFR, and cytokeratin 5/6 indicate a more specific definition of basal-like breast cancer, mostly TNBC, which better predicts breast cancer survival [5]. Cheang also showed that the core basal group has 1.62 times greater risk for breast cancer-specific death, whereas the non-core basal group does not have a clinically significant risk [5]. Siziopikou reported that because the majority of TNBC patients express EGFR, the subgroup may derive benefit from EGFR-targeted therapies [11].

\section{Conclusion}

In conclusion, our study indicates that TNBC tends to display a worse clinical course. Notably in node-positive patients, TNBC does play a prognostic role in overall survival. In Taiwan, new strategies of chemotherapy and targeted therapy should be investigated for patients with TNBC.

\section{Competing interests}

The authors declare that they have no competing interests.

\section{Authors' contributions}

CL designed the concept of this study, drafted the manuscript and performed treatment. HST, LSC and SJK collected the data and performed the statistical analysis. TWC approved the final manuscript. DRC designed the concept of this study and provided treatment coordination. All authors read and approved the final manuscript.

\section{Acknowledgements}

We thank the personnel at the Cancer Registry, Changhua Christian Hospital, for their great help.

\section{References}

I. Alexe G, Dalgin GS, Scanfeld D, Tamayo P, Mesirov JP, Ganesan S, Delisi C, Bhanot G: Breast cancer stratification from analysis of micro-array data of micro-dissected specimens. Genome Inform 2007, 18: I30-140.

2. Horwitz KB, Dye WW, Harrell JC, Kabos P, Sartorius CA: Rare steroid receptor-negative basal-like tumorigenic cells in luminal subtype human breast cancer xenografts. Proc Natl Acad Sci USA 2008, 105:5774-5779.

3. Liedtke C, Mazouni C, Hess KR, Andre F, Tordai A, Mejia JA, Symmans WF, Gonzalez-Angulo AM, Hennessy B, Green M, et al.: 
Response to neoadjuvant therapy and long-term survival in patients with triple-negative breast cancer. J Clin Oncol 2008, 26: |275-|28|.

4. Yin WJ, Lu JS, Di GH, Lin YP, Zhou LH, Liu GY, Wu J, Shen KW, Han QX, Shen ZZ, Shao ZM: Clinicopathological features of the triple-negative tumors in Chinese breast cancer patients. Breast Cancer Res Treat 2009, I I 5(2):325-33.

5. Cheang MC, Voduc D, Bajdik C, Leung S, McKinney S, Chia SK, Perou CM, Nielsen TO: Basal-like breast cancer defined by five biomarkers has superior prognostic value than triple-negative phenotype. Clin Cancer Res 2008, 14:1368-1376.

6. Mersin H, Yildirim E, Berberoglu U, Gulben K: The prognostic importance of triple negative breast carcinoma. Breast 2008, 17:34I-346.

7. Nguyen PL, Taghian AG, Katz MS, Niemierko A, Abi Raad RF, Boon WL, Bellon JR, Wong JS, Smith BL, Harris JR: Breast cancer subtype approximated by estrogen receptor, progesterone receptor, and HER-2 is associated with local and distant recurrence after breast-conserving therapy. J Clin Oncol 2008, 26:2373-2378.

8. Rakha EA, Reis-Filho JS, Ellis IO: Basal-like breast cancer: a critical review. J Clin Oncol 2008, 26( I5):2568-8I.

9. Mehta RS: Dose-dense and/or metronomic schedules of specific chemotherapies consolidate the chemosensitivity of triple-negative breast cancer: a step toward reversing triplenegative paradox. J Clin Oncol 2008, 26:3286-3288. author reply 3288

10. Rakha EA, El-Sayed ME, Green AR, Lee AH, Robertson JF, Ellis IO: Prognostic markers in triple-negative breast cancer. Cancer 2007, 109:25-32.

II. Siziopikou KP, Ariga R, Proussaloglou KE, Gattuso P, Cobleigh M: The challenging estrogen receptor-negative/progesterone receptor-negative/HER-2-negative patient: a promising candidate for epidermal growth factor receptor-targeted therapy? Breast J 2006, 12:360-362.

12. Kurebayashi J, Moriya T, Ishida T, Hirakawa H, Kurosumi M, Akiyama F, Kinoshita T, Takei H, Takahashi K, Ikeda M, Nakashima K: The prevalence of intrinsic subtypes and prognosis in breast cancer patients of different races. Breast 2007, I6(Suppl 2):S72-77.

13. Nishimura $R$, Arima $N$ : Is triple negative a prognostic factor in breast cancer? Breast Cancer 2008, I 5:303-308.

14. Haffty BG, Yang Q, Reiss M, Kearney T, Higgins SA, Weidhaas J, Harris $L$, Hait $W$, Toppmeyer $D$ : Locoregional relapse and distant metastasis in conservatively managed triple negative earlystage breast cancer. J Clin Oncol 2006, 24:5652-5657.

15. Perreard L, Fan C, Quackenbush JF, Mullins M, Gauthier NP, Nelson E, Mone M, Hansen H, Buys SS, Rasmussen K, et al: Classification and risk stratification of invasive breast carcinomas using a real-time quantitative RT-PCR assay. Breast Cancer Res 2006, 8:R23.

16. Kim MJ, Ro JY, Ahn SH, Kim HH, Kim SB, Gong G: Clinicopathologic significance of the basal-like subtype of breast cancer: a comparison with hormone receptor and Her2/neu-overexpressing phenotypes. Hum Pathol 2006, 37:1217-1226.

17. Fulford LG, Reis-Filho JS, Ryder K, Jones C, Gillett CE, Hanby A, Easton D, Lakhani SR: Basal-like grade III invasive ductal carcinoma of the breast: patterns of metastasis and long-term survival. Breast Cancer Res 2007, 9:R4.

18. Carey LA, Perou CM, Livasy CA, Dressler LG, Cowan D, Conway K, Karaca G, Troester MA, Tse CK, Edmiston S, et al:: Race, breast cancer subtypes, and survival in the Carolina Breast Cancer Study. JAMA 2006, 295:2492-2502.

19. Dent R, Trudeau M, Pritchard KI, Hanna WM, Kahn HK, Sawka CA, Lickley LA, Rawlinson E, Sun P, Narod SA: Triple-negative breast cancer: clinical features and patterns of recurrence. Clin Cancer Res 2007, I 3:4429-4434.

20. Langerod A, Zhao H, Borgan O, Nesland JM, Bukholm IR, Ikdahl T, Karesen R, Borresen-Dale AL, Jeffrey SS: TP53 mutation status and gene expression profiles are powerful prognostic markers of breast cancer. Breast Cancer Res 2007, 9:R30.

21. Rhee J, Han SW, Oh DY, Kim JH, Im SA, Han W, Park IA, Noh DY, Bang YJ, Kim TY: The Clinicopathologic Characteristics and Prognostic Significance of Triple-Negativity in Node-Negative Breast Cancer. BMC Cancer 2008, 8:307.

22. Ryden L, Landberg G, Stal O, Nordenskjold B, Ferno M, Bendahl PO: HER2 status in hormone receptor positive premenopausal primary breast cancer adds prognostic, but not tamoxifen treatment predictive, information. Breast Cancer Res Treat 2008, 109:35I-357.

23. Kim HJ, Cui X, Hilsenbeck SG, Lee AV: Progesterone receptor loss correlates with human epidermal growth factor receptor 2 overexpression in estrogen receptor-positive breast cancer. Clin Cancer Res 2006, 12:1013s-1018s.

\section{Pre-publication history}

The pre-publication history for this paper can be accessed here:

http://www.biomedcentral.com/1471-2407/9/192/pre pub
Publish with Bio Med Central and every scientist can read your work free of charge

"BioMed Central will be the most significant development for disseminating the results of biomedical research in our lifetime. "

Sir Paul Nurse, Cancer Research UK

Your research papers will be:

- available free of charge to the entire biomedical community

- peer reviewed and published immediately upon acceptance

- cited in PubMed and archived on PubMed Central

- yours - you keep the copyright

Submit your manuscript here:

http://www.biomedcentral.com/info/publishing_adv.asp 